\title{
Palentiella palentina n.g. n.sp. (BRAQUIÓPODO CAMAROTOECHIOIDEA) DEL EIFELIENSE SUPERIOR DE LA REGIÓN PALENTINA (CORDILLERA CANTÁBRICA ORIENTAL, N DE ESPAÑA)
}

\author{
Jenaro L. GARCÍA-ALCALDE \\ Departamento de Geología (Área Paleontología), c/ Jesús Arias de Velasco, \\ s/n, 33005 Oviedo (Asturias). email: jalcalde@geol.uniovi.es
}

García-Alcalde, J. L. 2009. Palentiella palentina n.g. n.sp. (braquiópodo Camarotoechioidea) del Eifeliense Superior de la Región Palentina (Cordillera Cantábrica Oriental, N de España). [Palentiella palentina n.g. n.sp. (Camarotoechioidea Brachiopoda) from the Upper Eifelian of the Palentian Region (Eastern Cantabrian Mountains, N Spain).] Revista Española de Paleontología, 24 (1), 79-92. ISSN 0213-6937.

\begin{abstract}
A new upper Eifelian (Middle Devonian) camarotoechioid Septalariidae, Palentiella palentina n.g. n.sp., from the Palentian domain (eastern Cantabrian Mountains, N Spain) is described. Two other upper Eifelian species, the Moroccan "Camarotoechia" willefertae Drot, 1971, and the Czech Nemesa hertae Havlíček, 1961, are also included in the new genus. All the three species mentioned above appeared during the faunal renewal following the Choteč-jugleri Event (lower Eifelian), but none of them got beyond the further Kačák-otomari Event (uppermost Eifelian). P. palentina is an excellent stratigraphical guide in the Devonian of the Palentian domain, where it occurs everywhere in the black shale-nodular marly limestone alternances of the Gustalapiedra Formation, inmediately below the Man Member.
\end{abstract}

Keywords: Articulated brachiopods Septalariidae, Eifelian, Palentian domain, Cantabrian Mountains, N Spain.

\section{RESUMEN}

Se describe un nuevo braquiópodo camarotoéquido Septalariidae, Palentiella palentina n.g. n.sp, del Devónico Medio (Eifeliense superior) del dominio Palentino de la Cordillera Cantábrica. Se incorporan al nuevo género, además de la especie-tipo, P. palentina, otras dos de la misma edad: "Camarotoechia" willefertae Drot, 1971, de Marruecos, y Nemesa hertae Havlíček, 1961, de la República Checa. Los taxones referidos se originaron en la fase de renovación faunística que siguió al Evento Choteč-jugleri, durante el Eifeliense inferior, pero ninguno de ellos consiguió superar el evento Kačák-otomari (Eifeliense terminal) posterior. P. palentina es una excelente guía estratigráfica en el Devónico del Dominio Palentino, donde aparece siempre acantonada en la alternancia de pizarras y calizas nodulosas margosas de la Formación Gustalapiedra, inmediatamente por debajo del Miembro Man.

Palabras clave: Braquiópodos articulados Septalariidae, Eifeliense, Dominio Palentino, Cordillera Cantábrica, norte de España.

\section{INTRODUCCIÓN}

La existencia de dos regiones paleogeográficas en el Devónico marino de la Cordillera Cantábrica, bien diferenciadas a partir del Emsiense, es conocida desde hace tiempo (Brouwer, 1964). Por un lado, el llamado Dominio Astur-Leonés, que abarca todos los afloramientos de
Asturias, la mayoría de los de León y buena parte de los de Palencia (Fig. 1), corresponde a ámbitos de plataforma, de escasa profundidad, propios de las Magnafacies Ardeno-Renana y Eifeliana (Erben, 1962). Por otro, el Dominio Palentino (Fig. 1), que comprende los afloramientos de la parte más septentrional de León y Palencia y de la oriental de Santander (Fig. 1), corresponde a ambientes de 
plataforma externa, relativamente más profundos, propios de la Magnafacies Herciniana o Bohemo-Herciniana (Erben, 1962). Las diferencias litológicas y paleontológicas entre ambos dominios son marcadas y dificultan su precisa correlación, aunque las líneas generales se conocen bien (García-Alcalde, 1996; García-Alcalde et al., 2002).

La superior extensión superficial y mejor accesibilidad de los afloramientos astur-leoneses frente a los palentinos provocó un desequilibrio histórico a favor del conocimiento relativo de aquéllos (García-Alcalde, 1998a). En lo que se refiere a estudios paleontológicos, máxime en los centrados en sucesiones del Devónico Medio y Superior, por ejemplo, hay una superioridad abrumadora de ensayos sobre la variada y abundante fauna nerítica astur-leonesa frente a la pelágica del Dominio Palentino. En particular, elementos bentónicos como los braquiópodos, omnipresentes en el Dominio Astur-Leonés, con frecuencia representados por poblaciones abundantes y bien conservadas, fueron amplia y minuciosamente tratados por investigado- res nacionales y extranjeros desde los trabajos pioneros de Verneuil \& d'Archiac (1845) y Prado \& Verneuil (1850), hace más de 150 años, mientras que en el Palentino, donde son siempre elementos escasos, dispersos y peor conservados, fueron tradicionalmente olvidados y tan sólo aparecen en referencias nominales de valor desigual y, en todo caso, relativo (Jahnke et al., 1983; García-Alcalde et al., 1988, 1990; Truyols-Massoni et al., 1990).

García-Alcalde (1999) abordó, con carácter previo, el problema dando información estratigráfica, paleogeográfica y paleoecológica sobre algunas especies de braquiópodos hercínicos del Devónico Medio del Dominio Palentino, incluyendo dibujos de las formas más representativas. Todos los taxones relacionados en dicho trabajo se acantonan en torno al llamado Miembro Man de la Formación Gustalapiedra (Figs. 2, 3 y 9). De acuerdo con la distribución estratigráfica de los elementos conocidos en otras áreas del mundo, su origen se encontraría en la fase de renovación faunística que siguió al Evento Choteč-jugleri (Walliser,
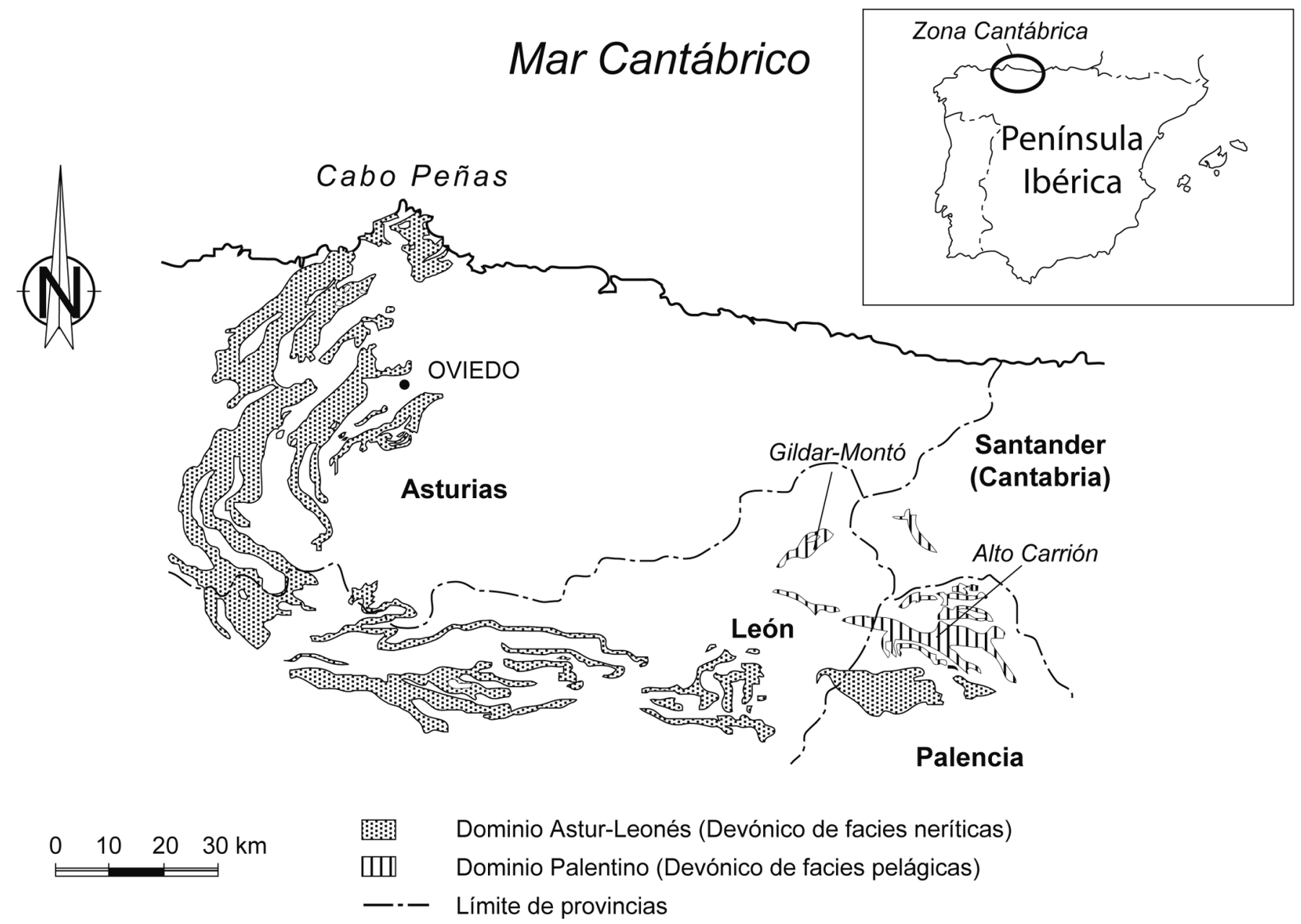

Figura 1. Dominios paleogeográficos del Devónico de la Cordillera Cantábrica (Zona Cantábrica) y situación de las áreas palentinas mencionadas en el presente trabajo.

Devonian paleogeographical domains in the Cantabrian Mountain (Cantabrian Zone) and setting of the Palentian areas mentioned herein. 


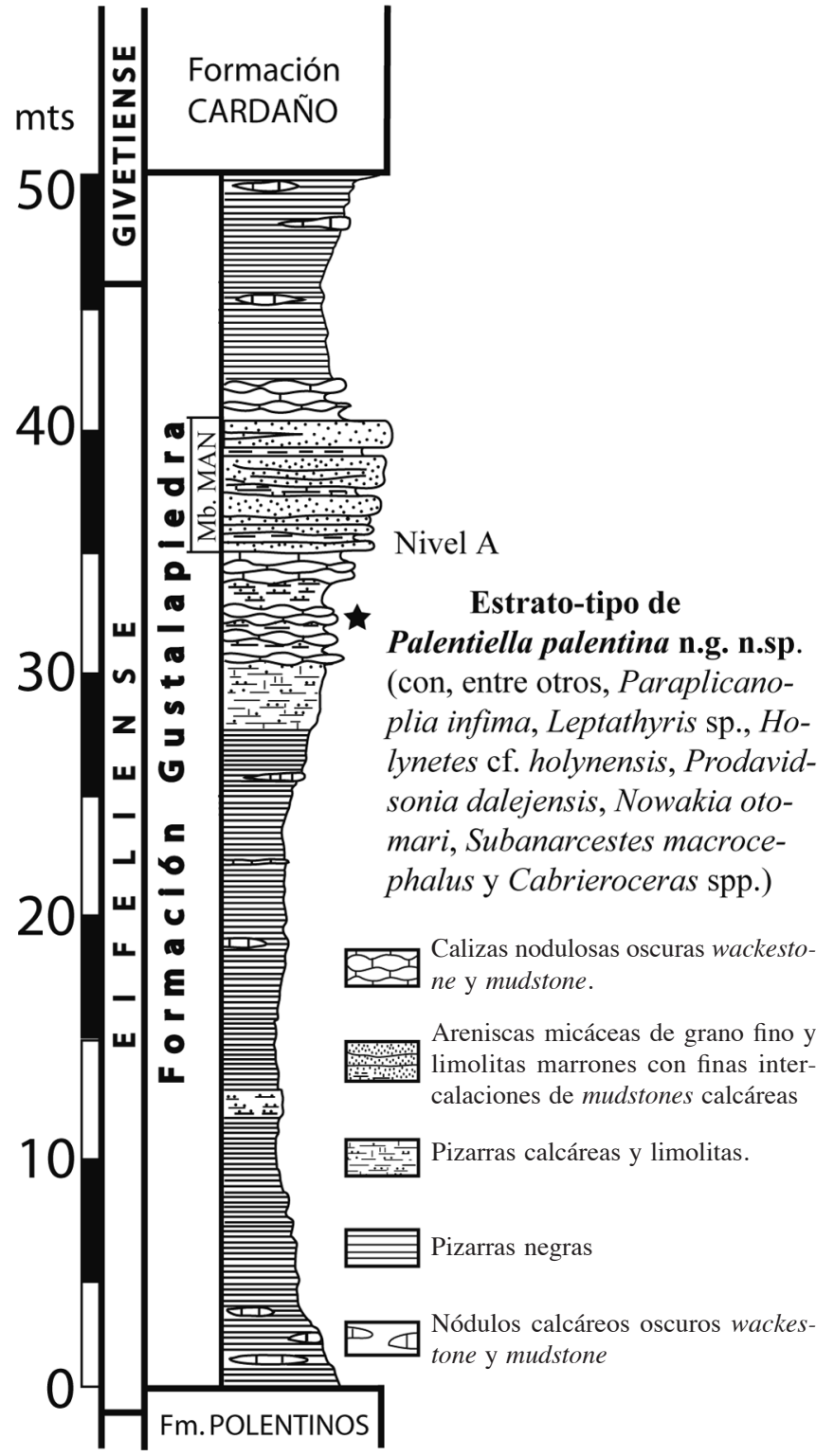

Figura 2. Columna estratigráfica de la Formación Gustalapiedra con situación del estrato-tipo de Palentiella palentina n.g. n.sp. y fauna pelágica acompañante de braquiópodos, dacrioconáridos y ammonoideos. Stratigraphical section of the Gustalapiedra Formation. Setting of Palentiella palentina n.g.n.sp. stratotype and accompanying pelagic fauna (brachiopods, dacryoconarids, and ammonoids).

1985; García-Alcalde, 1998b), pero todos desaparecieron víctimas de las alteraciones ambientales relacionadas con el Evento Kačák-otomari (Walliser, 1985; García-Alcalde, 1998b). Muchas de las formas citadas, de pequeño tamaño y concha ligera, como Leptathyris sp. (Fig. 4a-c), Holynetes cf. holynensis (Fig. 4d), Prodavidsonia dalejensis (Fig. 5a-c) y Paraplicanoplia infima (Fig. 5d), debieron ser epiplanctónicas y vivirían ligadas a cuerpos flotantes, masas de algas, por ejemplo. Pero otras, de tamaño medio, gruesas y mucho más pesadas, lo harían a nivel del fondo llevando una existencia precaria, en riesgo permanente de hundirse en el blando sustrato arcilloso circundante. Entre estas últimas se encuentra un interesante rinconélido camarotoéquido de la Familia Septalariidae Havlíček, 1960 , con características originales suficientes como para incluirlo en un género y especie nuevas, Palentiella palentina n.g. n.sp, cuya descripción detallada se aborda a continuación. La asociación de esta especie con dacrioconáridos como Nowakia otomari y ammonoideos como $\mathrm{Su}$ banarcestes macrocephalus y Cabrieroceras spp. dentro de la Biozona de conodontos de Tortodus kockelianus, indica una edad Eifeliense superior (García-Alcalde, 1998b). El nuevo taxón es próximo a formas citadas en capas de la misma edad en Bohemia, Alemania y Marruecos, en diferentes combinaciones sistemáticas, parte de las cuales pertenecería, incluso, al nuevo género (ver luego).

\section{SISTEMÁTICA}

\section{Superfamilia Camarotoechioidea Schuchert, 1929 Familia Septalariidae Havlíček, 1960}

\section{Palentiella n.gen.}

Especie-tipo: Palentiella palentina n.sp. (Devónico Medio, Eifeliense superior)

Diagnosis: Concha generalmente pequeña, dorsi-biconvexa, lisa, salvo cerca de las comisuras, donde aparece un número variable de costillas simples, bajas y redondeadas. Pliegue medio dorsal y seno ventral débiles, iniciándose en la mitad anterior de la concha. Comisura frontal invaginada y uniplegada; lengüeta alta; espinas marginales. Placas dentales inexistentes o muy menudas, no funcionales. Plataforma cardinal indivisa, deprimida medianamente, soportada en parte por un septo alto, agudo y largo. Apófisis cardinal pectinada, desarollada en la mitad posterior de la plataforma cardinal; crura próximos y paralelos.

Diagnosis: Shell medium size, dorsi-biconvex, with emarginated anterior commissure. Ribbed ornamentation developed only near the commissures, and constituted by a variable number of simple, low, rounded ribs. Faint dorsal median fold and ventral sinus, beginning at mid-length. Uniplicate anterior commissure. High tongue. Marginal spines. Minute, non-functional dental plates or lacking. Undivided, medially depressed hinge-plate, partially supported by a long, high, sharp median septum. Pectinate cardinal processus, developed in the mid, posterior part of the hinge plate. Parallel and closely situated crural processus.

Discusión: La inclusión del nuevo género entre los Septalariidae se basa en la morfología general de la concha 
y, particularmente, en el desarrollo de apófisis cardinal y espinas marginales, raras o ausentes en los demás géneros de Camarotoechioidea, además del escaso, casi nulo, desarrollo de las placas dentales, característica frecuente entre los septaláridos y mucho menos en las otras Familias del Orden: Camarotoechiidae y Leiorhynchidae.

De los géneros de la Familia Septalariidae, Palentiella se parece, sobre todo, a Septalaria Leidhold, 1928, y a Pseudocamarophoria Wedekind, 1926, ambos del Emsiense al Givetiense. Septalaria, fue objeto de varias revisiones que permitieron la reasignación de muchas de sus especies a otros géneros (Amissopecten, Pseudocamarophoria, Onugorhynchia, etc), pero requeriría una revisión ulterior. La especie-tipo de Septalaria, es casi equidimensional, con ángulo apical en torno a $90^{\circ}$, pliegue medio dorsal, seno ventral y ornamentación costada iniciándose en la región umbonal, con el seno ocupando en el frente más del $60 \%$ de la anchura de la concha, campo muscular ventral débilmente impreso y placas dentales cortas pero evidentes. Las especies incluidas en Palentiella, en cambio, son claramente más anchas que largas, con ángulo apical en torno a $120^{\circ}$, pliegue medio dorsal y seno ventral desarrollados sólo a partir de la mitad de la longitud y costillas presentes únicamente cerca o en la misma comisura y en la lengüeta ventral, el campo muscular ventral fuertemente excavado en el fondo de la valva y placas dentales casi inapreciables. Algunas especies asignadas a Septalaria, como S. palumbina (Barrande, 1879), son mucho más anchas que largas y tienen ángulo apical muy abierto, pero difieren claramente de las especies de Palentiella, en el mayor desarrollo de la ornamentación costada, que comienza casi en los ápices de las valvas, y en la posesión de placas dentales bien desarrolladas, y carecen, además, del engrosamiento posterior y excavamiento del campo muscular del nuevo género. Pseudocamarophoria Wedekind, 1926, se parece en el contorno y estructuras dorsales, pero tiene perfil lenticular, las costillas, pliegue medio y seno ventral se extienden desde la región umbonal, las placas dentales son más desarrolladas que las de Palentiella, y carece de apófisis cardinal.

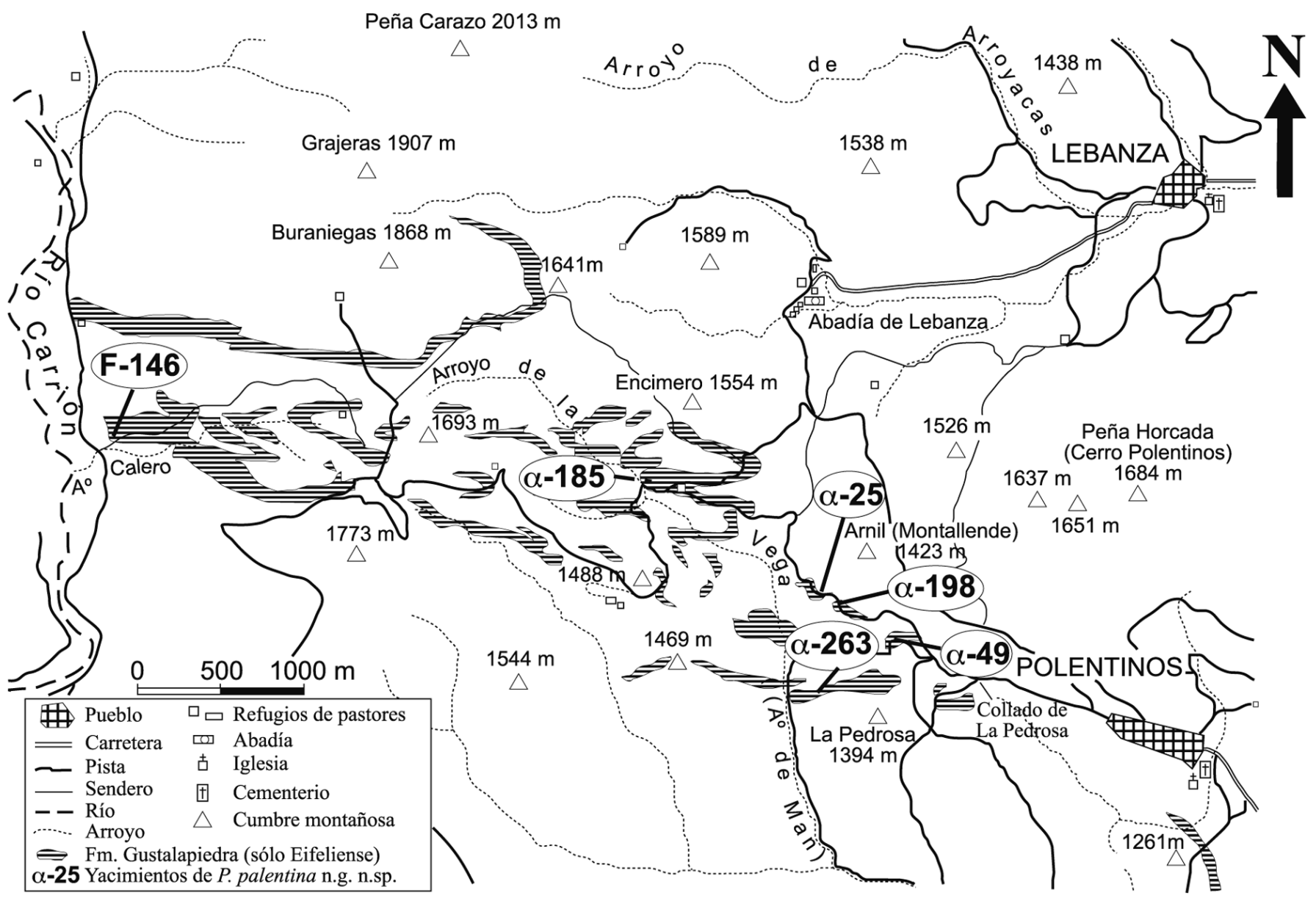

Figura 3. Afloramientos de la Formación Gustalapiedra (sólo la parte Eifeliense) en el área del Alto Carrión y situación de los principales yacimientos de Palentiella palentina n.g. n.sp.

Gustalapiedra Formation (only the Eifelian part) outcrops in the Alto Carrion area, and setting of the main localities of Palentiella palentina n.g. n.sp. 


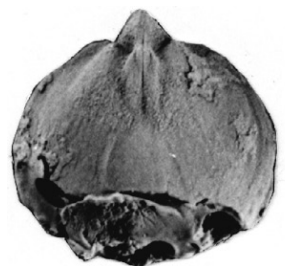

a1

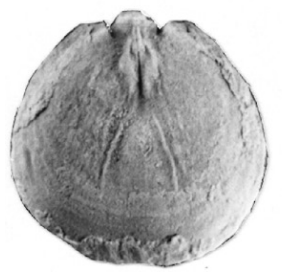

b1

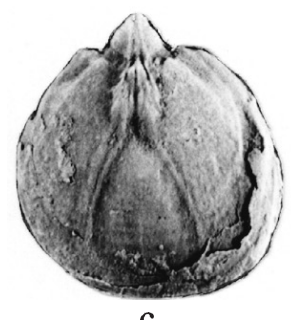

c

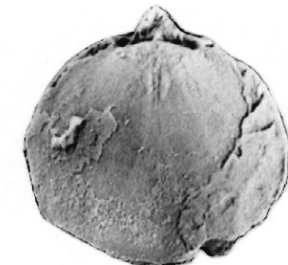

a2

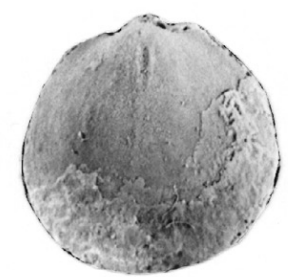

b2 a3

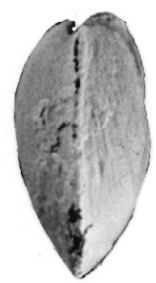

b3
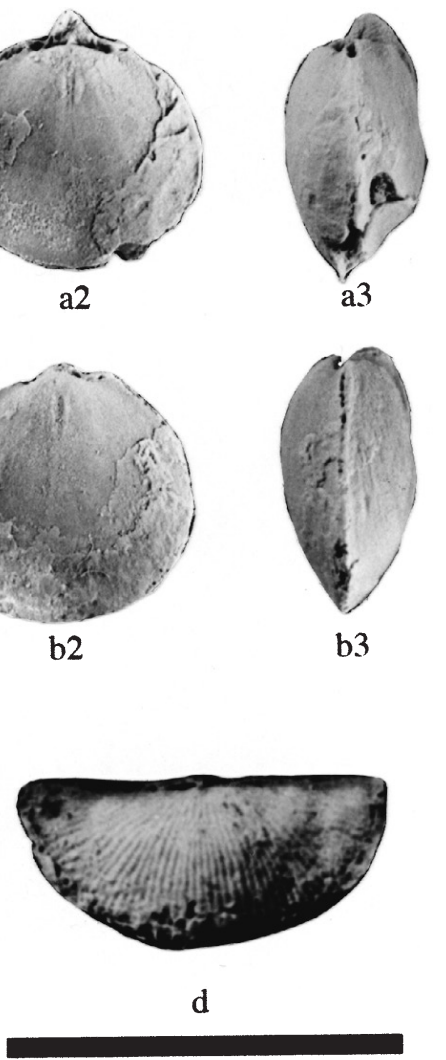

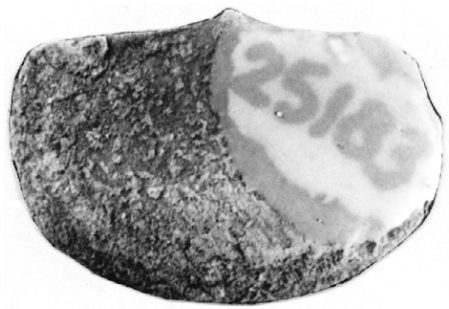

a1

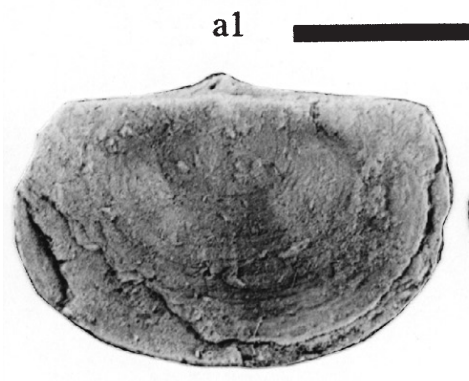

a2

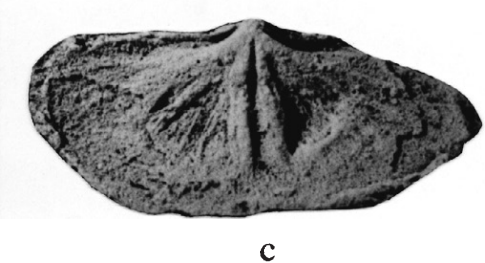

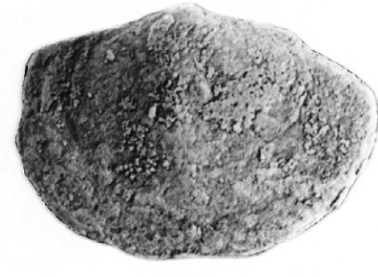

b1

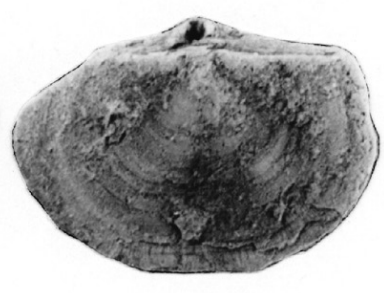

b2

Figura 4. a-c: Leptathyris sp. (moldes internos completos), yacimiento $\alpha-25$, pista de la abadía de Lebanza a Polentinos, $3 \mathrm{~m}$ bajo las areniscas del Mb. Man de la Fm. Gustalapiedra, Eifeliense superior. a1-a3: DPO 34100, vistas ventral, dorsal y lateral. b1-b3: DPO 34101, vistas ventral, dorsal y lateral. c: DPO 34099, vista ventral. d; Holynetes cf. holynensis Havlíček \& Racheboeuf, 1979. DPO 25181, molde externo dorsal (kop. García-Alcalde et al., 1990, fig. 9.8, con mayor aumento), yacimiento. $\alpha-263$, localidad típica del Mb. Man, Eifeliense superior. Barras negras: $1 \mathrm{~cm}$; izquierda para las figuras a-c (Leptathyris); derecha: para d (Holynetes).

$\boldsymbol{a}-\boldsymbol{c}$ : Leptathyris sp. (complete internal moulds), $\alpha-25$, track from the Abadia de Lebanza to Polentinos, 3 $m$ below the sandstones of the Man Mb of the Gustalapiedra Fm, upper Eifelian. a1-a3: DPO 34100, ventral, dorsal and lateral views. b1-b3: DPO 34101, ventral, dorsal, and lateral views. c: DPO 34099, ventral view. d: Holynetes $c f$. holynensis Havliček \& Racheboeuf, 1979. DPO 25181, external dorsal mould (kop. García-Alcalde et al., 1990, fig. 9.8, additionally enlarged), $\alpha$-263. Type locality of the Man Mb, upper Eifelian. Black bars: $1 \mathrm{~cm}$; left for a-c (Leptathyris), right for $\boldsymbol{d}$ (Holynetes).

La especie-tipo de Nemesa Schmidt, 1941, N. nemesana Schmidt, 1941, es débilmente transversa, con ángulo apical en torno a $100^{\circ}$ y superficie lisa salvo por la presencia de una ancha elevación media ventral, que arranca

Figura 5. a-c: Prodavidsonia dalejensis Havlíček, 1956. a1-a2: DPO 25183 (nuevas fotografías del ejemplar figurado en García-Alcalde et al., 1990, figs. 9.18-19, aquí con mayor aumento), yacimiento $\alpha-263$ (ver antes), vistas ventral y dorsal. b1-b2: DPO 34089, yac. F-146, arroyo de El Calero cerca de su confluencia con el río Carrión, Fm. Gustalapiedra, Eifeliense superior, vistas ventral y dorsal. c: DPO 25184 (ejemplar figurado en García-Alcalde et al., 1990 Fig. 9.20, como Quasidavidsonia cf. vicina, aquí con mayor aumento), yac. $\alpha$-263 (ver antes), molde interno ventral. d: Paraplicanoplia infima (Havlíček, 1977). DPO 25185 (kop. García-Alcalde et al., 1990, fig. 9.22, más aumentada), molde interno ventral, yac. $\alpha-263$ (ver antes). Barra negra superior: $1 \mathrm{~cm}$, para a-c (P. dalejensis). Barra negra inferior: $1 \mathrm{~mm}$, sólo para d (P. infima). a-c: Prodavidsonia dalejensis Havlíček, 1956, a1-a2: DPO 25183 (new pictures of the García-Alcalde et al., 1990, figs. 9.18-19 figured specimen, additionally enlarged), locality $\alpha-263$ (see above), ventral, and dorsal views. b1-b2: DPO 34089, locality F-146, El Calero Creek, near junction with the Carrion River. Gustalapiedra Fm, upper Eifelian, ventral, and dorsal views. c: DPO 25184 (kop. specimen represented as Quasidavidsonia $c f$. vicina in García-Alcalde et al., 1990, Fig. 9.20, additionally enlarged), loc. $\alpha-263$ (see above), ventral internal mould. $d$ : Paraplicanoplia infima (Havlíček, 1977). DPO 25185 (kop. García-Alcalde et al., 1990, fig. 9.22, additionally enlarged), loc. $\alpha$-263 (see above), ventral internal mould. Upper black bar: $1 \mathrm{~cm}$, for a-c (P. dalejensis). Lower black bar: $1 \mathrm{~mm}$, only for $\boldsymbol{d}$ (P. infima). 


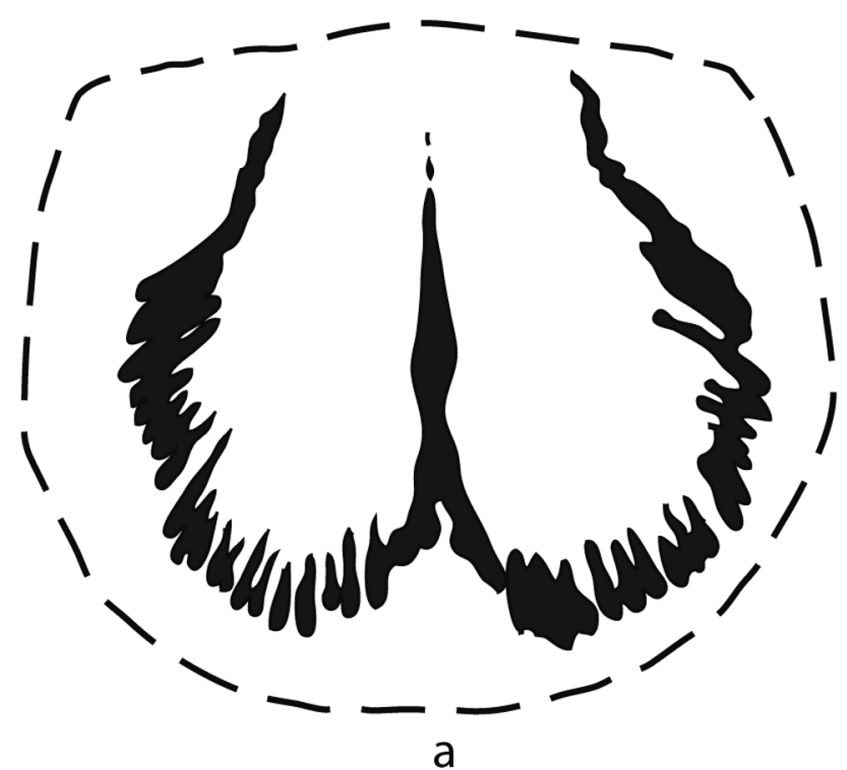

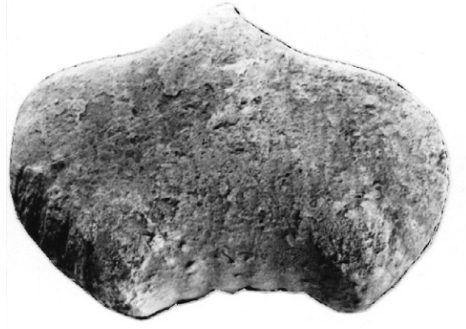
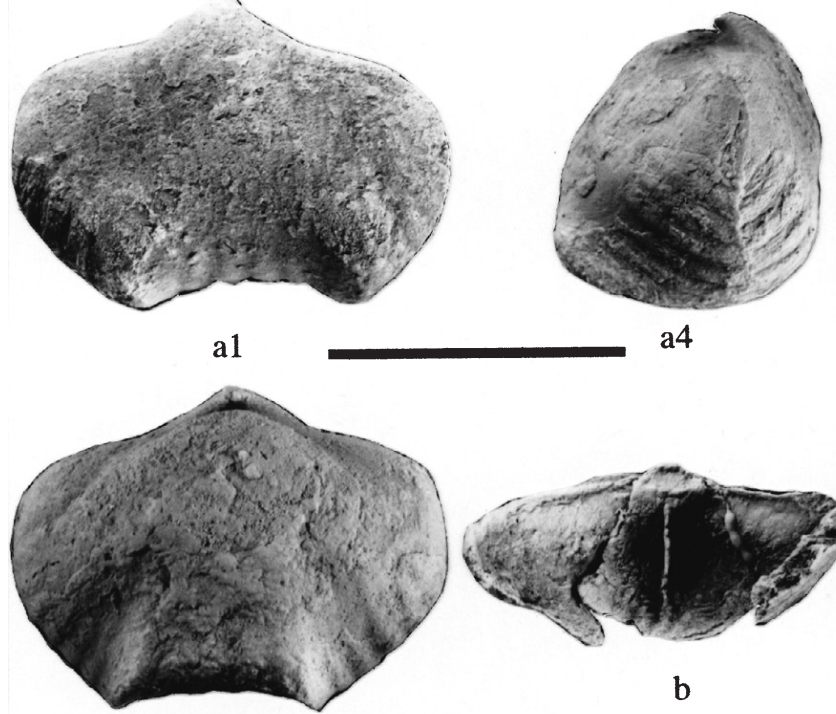

a2

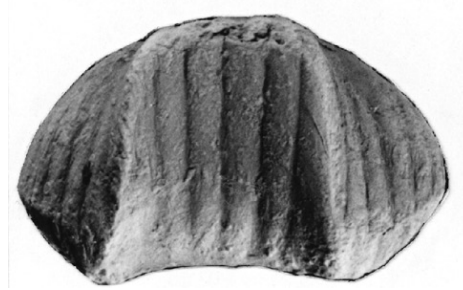

a3

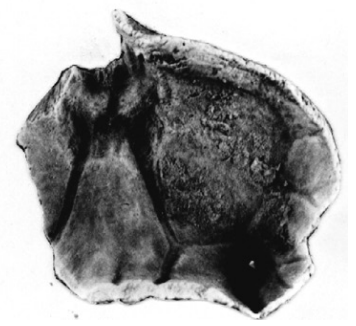

c

Figura 7. Palentiella palentina n.g. n.sp. a1-a4: Holotipo DPO 34089 , yacimiento $\alpha$-49bis, $3 \mathrm{~m}$ por debajo de las areniscas del Mb. Man, Eifeliense superior, vistas ventral, dorsal, anterior y lateral. b: Paratipo DPO 33420 , misma procedencia anterior, interior de valva dorsal fragmentaria. c: DPO 33403, yacimiento $\alpha$-263 (ver antes), interior de valva ventral fragmentaria. Barra negra: $1 \mathrm{~cm}$.

Palentiella palentina n.g. n.sp.a1-a4: Holotype DPO 34089 , locality $\alpha$-49bis, $3 \mathrm{~m}$ below the Man Mb sandstones, upper Eifelian, ventral, dorsal, anterior, and lateral views. b: Paratype DPO 33420, same locality as before, fragmentary dorsal valve interior. $\boldsymbol{c}$ : DPO 33403, locality $\alpha-263$ (see above), fragmentary ventral valve interior. Black bar: $1 \mathrm{~cm}$. rior ventral DPO 25186 (b) (algo idealizados), yacimiento $\alpha$-263, Eifeliense superior.

Paraplicanoplia infima (Havlíček, 1977). Drawings of an internal dorsal mould DPO 34105 (a), and of a ventral exterior DPO 25186 (b) (slightly idealized); locality $\alpha$-263, upper Eifelian.

del área umbonal y convierte la comisura anterior en surcoplegada. Sin embargo, una de las especies asignadas al taxón, $N$. hertae Havlíček, 1961, se aleja de la forma anterior en que es bastante más transversa, con ángulo apical ampliamente abierto y costillas numerosas, desarrolladas cerca de las comisuras de la concha y bastante débiles en

los flancos. Todas esas características son más bien propias de Palentiella n.g. donde se reacomoda dicha especie.

La especie descrita por Drot (1971) como “Camarotoechia" willefertae muestra las características propias de Palentiella n.g.: contorno semi-elíptico, netamente transverso, ángulo apical muy abierto, seno ventral y pliegue medio desarrollados por delante de la región umbonal, costillas desarrollados sólo cerca de las comisuras, muy débiles en los flancos, grandes espinas marginales, placas dentales poco desarrolladas y valva ventral engrosada posteriormente, con campo muscular muy excavado. Y aunque la autora de la especie señaló, con cierta vacila- 
ción, la ausencia de apófisis cardinal ("Vraisemblablement pas de processus cardinal, mais un très fort épaississement coquillier masquant le plateau cardinal qui apparaît par transparence", op. cit: 92), en nuestra opinión, el aludido "épaississement coquillier" es una verdadera apófisis cardinal que muestra además laminillas verticales, como se ve en las secciones seriadas de la Fig. text. 7 (Drot, op. cit.). Además, en varios ejemplares de una colección relativamente abundante (12 individuos) recogida por nosotros mismos en Marruecos, en una de las localidades citadas por J. Drot (Niveles TM 126/1 y 126/2, Tamjout-Ouihlane) e identificadas por la propia autora de la especie como "Camarotoechia" willefertae, se aprecia la existencia de espesamientos cardinales en la parte posterior de la valva dorsal que corresponden, sin duda, a apófisis cardinales. Por las razones enumeradas, incluimos también la especie de Drot entre las propias del nuevo género Palentiella.

Especies asignadas: Además de la especie-tipo, se incorporan al nuevo género, como acaba de razonarse, Nemesa hertae Havlíček, 1961, del Eifeliense de Bohemia, calizas de Hlubocepy, y "Camarotoechia" willefertae Drot, 1971, del Eifeliense superior o Givetiense basal de Marruecos, calizas nodulosas negras, con Cabrieroceras crispiforme, superpuestas a calizas encriníticas con Pinacites jugleri.

\section{Palentiella palentina n. gen. n.sp.} Figs $7,8,10-12$

v. 1990 Camarotoechia cf. willefertae; García-Alcalde et al., Fig. text. 7.

v. 1999 Palentiella pallantiana nom.nud.; García-Alcalde, p. 600, Figs. 2-4, Tab. 1.

Derivatio nominis: Procedente de la provincia de Palencia, N de España.

Material: 39 ejemplares. Holotipo DPO 34089 (Fig. 7.a1-a4), 9 Paratipos DPO 33416, 33417 (Fig. 8.b1-b4), 33420 (Fig. 7.b), 34088, 34090 (Fig. 12.b), 34091-34094, y otros 6 ejemplares DPO 33418-33419, 34089 y 39484-39486, procedentes del estrato y localidad típicos. 15 ejemplares DPO 25171 (Fig. 8.a1-a4), 33402 (Fig. 12.a), 33403 (Figs. 7.c y 11), 33404-33410, 33411 (Fig. 8.c1-c4), 33412-33414 y 34095 (Fig. 8.f1-f4), procedentes del estrato tipo del Miembro Man de la Fm Gustalapiedra, yacimiento $\alpha-263$ (Fig. 3), Eifeliense superior. 2 ejemplares, DPO 33415 y 34096 , del yacimiento $\alpha-25$ (Fig. 3), pizarras y lentejones de calizas nodulosas de la Fm. Gustalapiedra por debajo del Mb Man, Eifeliense superior. 6 ejemplares, DPO 33421 (Fig. 8.g1-g4), 33422-33426 procedentes del corte de la Fm. Gustalapiedra (Eifeliense superior), a lo largo de un tributario por la derecha del arroyo de El Cable (región del Gildar-Montó, Fig. 1), en las inmediaciones de una cabaña de pastores.

Todo el material está depositado en la Colección del Departamento de Geología, sección Paleontología (DPO), de la Universidad de Oviedo.
Localidad y estrato típicos: Pista que baja del collado de La Pedrosa (1,2 km al ONO del pueblo de Polentinos, $\mathrm{N}$ de Cervera de Pisuerga, Palencia) hacia el arroyo de La Vega (también llamado arroyo de Man o Monderrío), rama oriental de un tramo de la pista que describe una $U$ asimétrica invertida, cota 1.310 m y coordenadas $42^{\circ} 56^{\prime} 44^{\prime \prime}$ Lat. N, y $4^{\circ} 32$ ' 40" Long. O, del Mapa Topográfico Nacional E=1:25.000, hoja 106-II (32-13), Polentinos (Figs. 1, 3 y 9). Pizarras oscuras con lentejones de calizas nodulosas intercaladas, $3 \mathrm{~m}$ por debajo del primer paquete limolítico marrón (nivel A) de las areniscas y limolitas del Miembro Man de la Fm Gustalapiedra, Eifeliense superior (Fig. 2), Biozonas de dacrioconáridos Nowakia otomari y de conodontos Tortodus kockelianus (García-Alcalde et al., 1990), Yacimiento $\alpha$-49bis (Figs. 2-3 y 9). El nivel se sigue sobre la superficie de la pista y en su margen septentrional, en el ribazo derecho de un arroyuelo, habitualmente seco. El afloramiento de la Fm. Gustalapiedra pertenece al autóctono relativo de una ventana tectónica, cabalgada al $\mathrm{N}$ y al $\mathrm{S}$ por pizarras de la parte alta de la Fm. Abadía (Emsiense superior) (Fig. 9) y, consecuentemente, se encuentra bastante tectonizado.

Diagnosis: Concha de tamaño medio, contorno subpentagonal, más ancha que larga $\left(\mathrm{a} / \mathrm{L}_{\text {med }}=1,36\right)$, fuertemente dorsi-biconvexa (valva dorsal aproximadamente 2 veces más alta que la ventral). Margen anterior invaginado. Gancho pequeño, erecto a curvado, perforado por un foramen menudo y circular, mesotírido a epitírido, limitado basalmente por un deltidio fuerte y convexo. Ángulo apical muy obtuso $\left(121^{\circ}\right.$ por término medio). Seno ventral débil, cóncavo a aplanado; lengüeta alta y rectangular, dirigida dorsal a posterodorsalmente. Espinas marginales finas y largas. Comisuras débilmente denticuladas. Pliegue medio dorsal bajo y aplanado. Costillas bajas y redondeadas, desarrollándose sólo cerca de las comisuras y a lo largo de la lengüeta ventral (20-26 costillas en total, $\mathrm{m}(4-6) /(3-5)$, a veces con una parietal, $1(7-9) /(8-10))$; espacios intercostales mucho más estrechos y agudos que las costillas. Región umbonal muy engrosada por depósitos conchíferos, excavados por el campo muscular ventral; euseptoide desarrollado por delante de las huellas musculares principales. Sistema de canales del manto embolsado (saccate), fuertemente impreso en el fondo de la valva. Dientes cardinales con dentículos secundarios; placas dentales muy pequeñas, no funcionales. Squama y glotta fuertes, bien desarrolladas.

Diagnosis: Medium size shell, sub-pentagonal outline, wider than long (average $a / L=1,36$ ), strongly dorsi-biconvex (dorsal valve ca. two times higher than the ventral). Anterior commissure emarginate. Small, erect to incurved beak; mesothyrid to epithyrid, circular, minute foramen; strong, convex deltidium. Very obtuse apical angle (average $121^{\circ}$ ). Faint, concave to flattened ventral sinus; high, rectangular, dorsal- to postero-dorsally directed tongue. Fine, long, marginal spines. Faintly denticulated commissures. Low, flattened median dorsal fold. Simple, anteriorly developed (near commissures and in the ventral tongue), low, roun- 

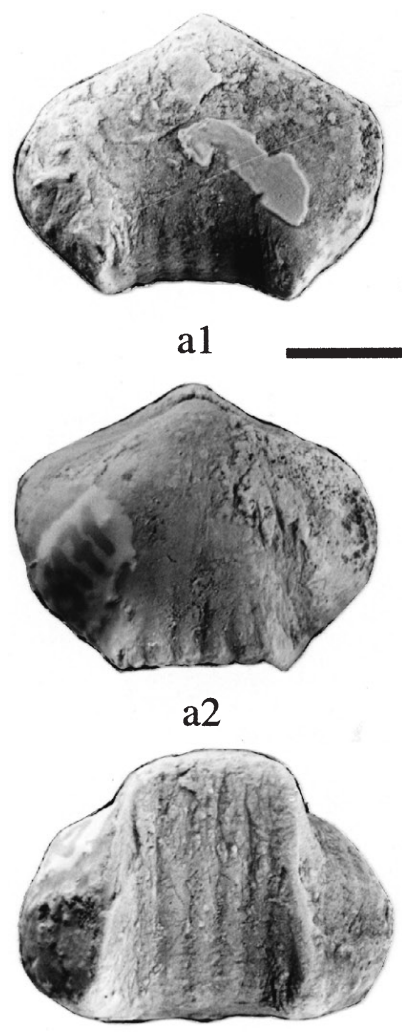

a3

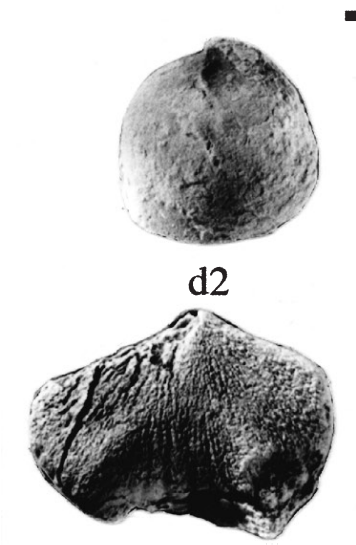

el

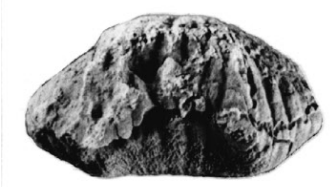

e2

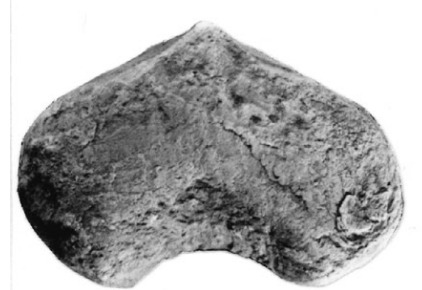

f1
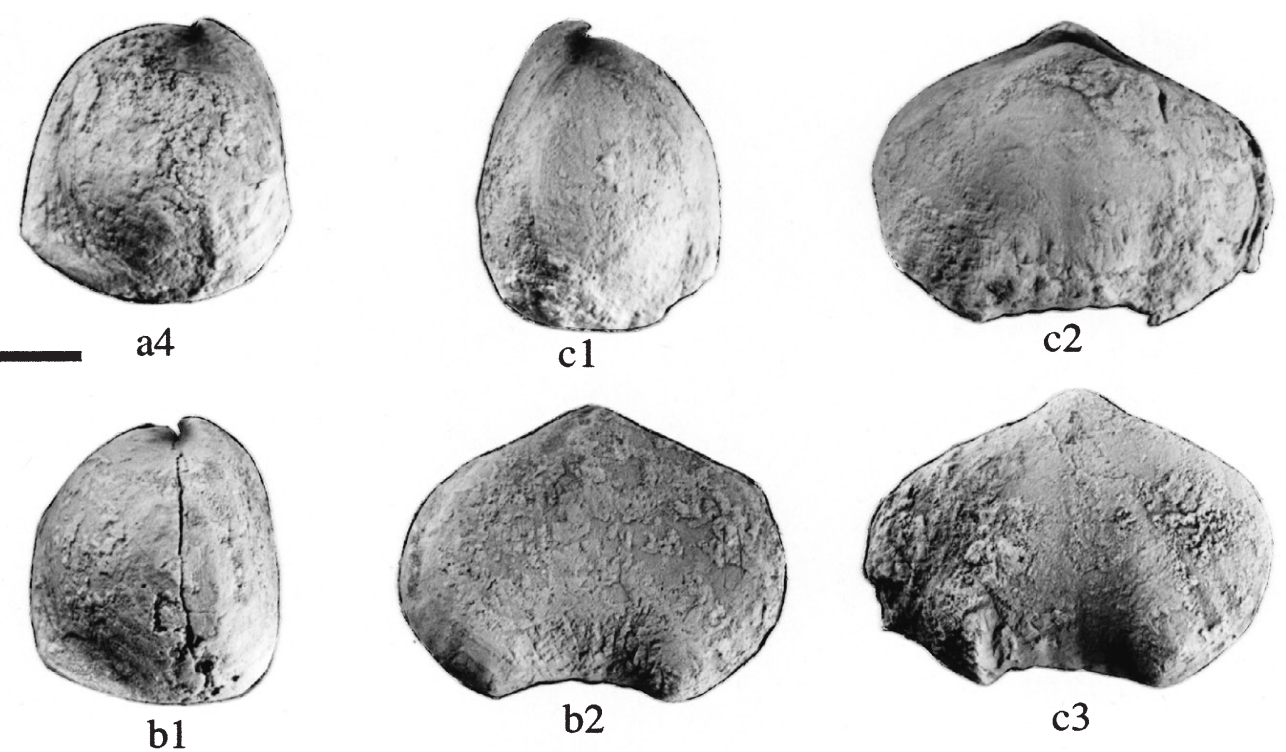

b2

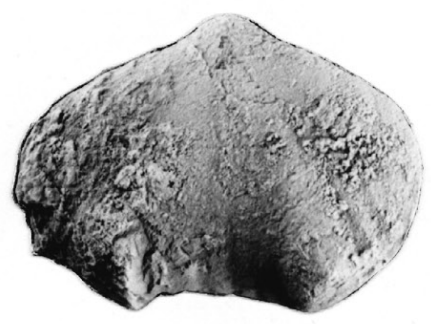

c3
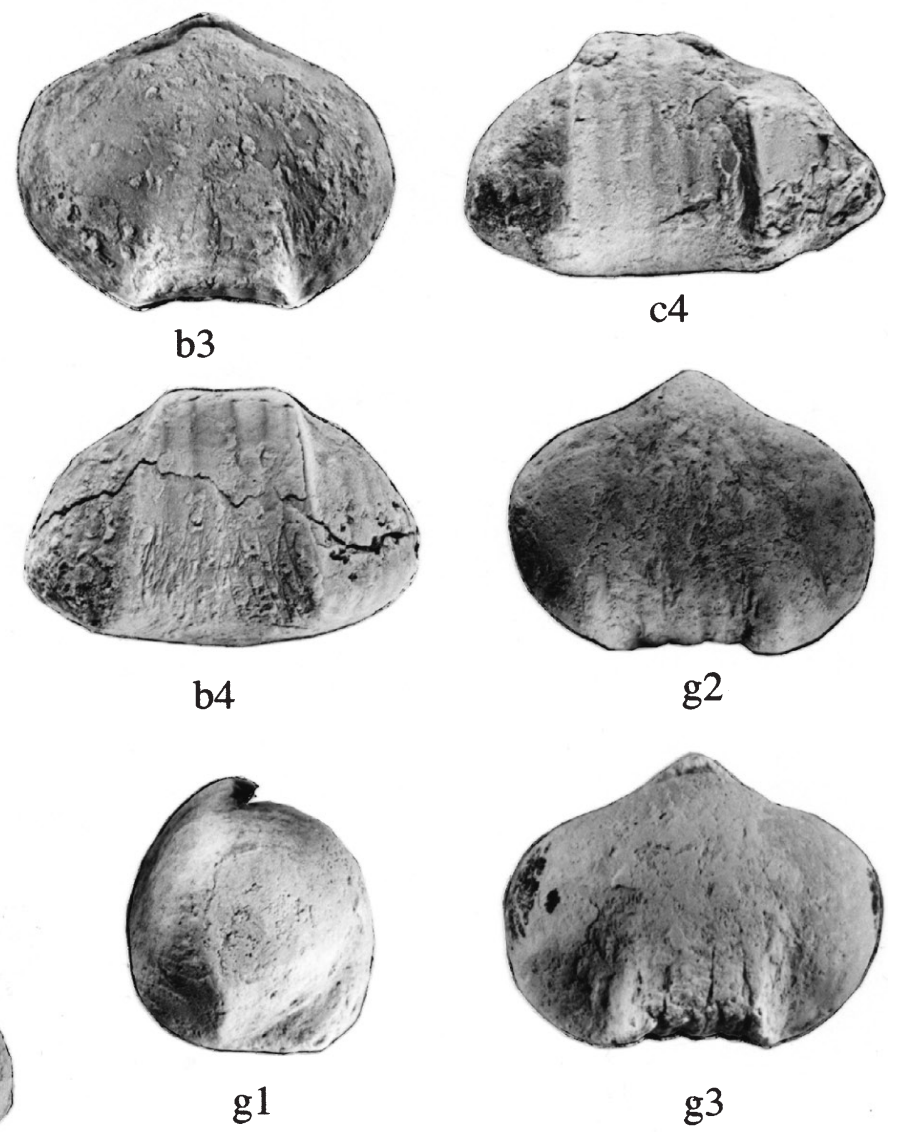

g3

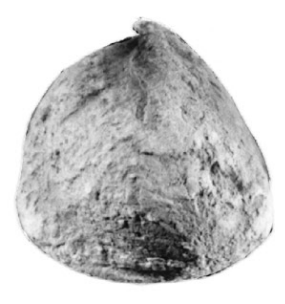

f4

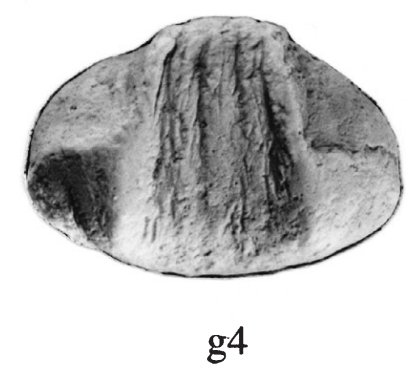


ded ribs, separated by much narrower, sharp interspaces (20-26 ribs, m(4-6)/(3,5), occassionally with one parietal rib, l(7-9)/(8-10)). Very thickened umbonal region. Ventral muscle field strongly excavated in the umbonal callus, with an euseptoidum anterior to the adductor-diductor scars. Strongly marked, saccate ventral mantle canals. Hinge teeth with secondary denticula; non-functional, minute dental plates. Well-developed squama and glotta.

Descripción: Concha de tamaño medio (L entre 8 y $13 \mathrm{~mm}$; $\mathrm{L}_{\text {med }}=10,5$, para 26 medidas; $\mathrm{a}_{\text {med }}=14,6$, para 26 medidas; $\mathrm{g}_{\text {med }}=16$, para 9 medidas), contorno subpentagonal, con el frente emarginado, más ancha que larga $\left(\mathrm{a} / \mathrm{L}_{\text {med }}=1,36\right.$, para 26 medidas $)$, con la máxima anchura situada algo por delante de la mitad de la longitud (por término medio a 0,56L, para 19 medidas), dorsibiconvexa, fuertemente inequivalva (valva dorsal unas 2 veces más alta que la ventral), globosa ( $\mathrm{g} / \mathrm{L}_{\mathrm{med}}=0,87$ para 16 medidas) (Fig. 10). La escasa correlación entre L y g (Fig. 10), refleja el hecho de que todos los ejemplares presentan un grado variable de aplastamiento diagenético. Ángulo apical obtuso, muy abierto $\left(\mathrm{a}_{\text {med }}=121^{\circ}\right.$, para 18 medidas). Seno ventral y pliegue medio dorsal desarrollados en el frente de la concha, iniciándose algo por delante de la mitad de la longitud (por término medio, hacia $0,54 \mathrm{~L}$, para 20 medidas). Seno de fondo débilmente cóncavo o aplanado, ocupando en el frente, por término medio, el $53 \%$ de la anchura de la concha. Pliegue medio dorsal bajo, de techo aplanado.

Valva dorsal en vista lateral fuertemente convexa en la región del umbo y regular y poco convexa a continuación, con la máxima altura en el frente o algo detrás, regularmente convexa en vista anterior, sin formar paries geniculatus individualizado. Valva ventral regularmente convexa, doblándose fuertemente en el frente de la concha para formar una lengüeta rectangular o casi, alta, de fondo aplanado, dirigida dorsal a postero-dorsalmente en su extremo distal; gancho ventral pequeño, erecto a incurvado, perforado por un menudo foramen apical mesotírido a epitírido, limitado en su base por un deltidio ancho y convexo. Aristas umbonales fuertes, delimitando áreas lunulares, cortas y estrechas, algo deprimidas. Bordes de ambas valvas con espinas marginales finas y largas. Las valvas se encuentran en las comisuras laterales según un ángulo obtuso, entre $90^{\circ}$ y $150^{\circ}$; comisuras débilmente indentadas por las costillas, la frontal uniplegada y las laterales algo desviadas en dirección ventral.

Superficie de la concha prácticamente lisa, salvo cerca de las comisuras laterales y a lo largo de la lengüeta, donde se desarrollan costillas simples, anchas, bajas y redondeadas, separadas por espacios intercostales mucho más estrechos y agudos; fórmula costal media 4-6/3-5, con costillas de anchura irregular y una costilla parietal apareciendo ocasionalmente; 8 a 10 costillas obsolescentes en cada flanco, visibles usualmente sólo cerca de la comisura.

La parte posterior de la concha está fuertemente engrosada, excavándose las huellas musculares de los aductores en el espeso macizo de concha secundaria; huellas de los aductores ventrales profundas, pequeñas, longitudinalmente ovaladas, rodeadas por las de los diductores, que son trapezoidales y también alargadas; las huellas de los diductores están separados por delante de las de los aductores por una profunda y estrecha hendidura que desemboca en un área trapezoidal estríada, que limita el campo muscular por delante y a un nivel algo inferior, dividida por un fino y corto miofragma medio (euseptoide), a ambos lados del cual aparecen sendas oquedades subcirculares, profundas (Figs. 7c y 11).

Angioglifo ventral embolsado (en forma de bolsa o saco, saccate) (Figs 7f, 11), con vasos surcando en profundidad la superficie interna de la concha. Vasos genitales (vascula genitalia), reniformes, estrechos y poco excavados, flanqueando las huellas de los diductores; vasos medios (vascula media, Fig. 11) largos y estrechos, partiendo del campo muscular y divergiendo hacia delante hasta llegar cerca del tercio anterior de la longitud, donde se vuelven uno hacia el otro tendiendo a converger débilmente hacia la comisura frontal; del punto donde cambia la dirección de los vasos medios, arrancan ramas laterales, que se dirigen lateral, posterior y postero-medianamente, más o menos paralelas al borde de la valva, hasta alcanzar las huellas de los vasos genitales. De las ramas laterales se desgajan varias ramas secundarias, que se dividen dos o más veces antes de alcanzar las comisuras.

Figura 8. a-d y f-g: Palentiella palentina n.g. n.sp. a1-a4: DPO 25171 (nueva fotografía, más ampliada, del ejemplar figurado como "Camarotoechia" willefertae Drot, in García-Alcalde et al., 1990, fig. 9.1-4), yacimiento $\alpha$-263 (ver antes), vistas ventral, dorsal, anterior y lateral. b1-b4: Paratipo DPO 33417, yac. $\alpha$-49bis (ver antes), vistas lateral, ventral, dorsal y anterior. c1-c4: DPO 33411, yac. $\alpha$-263 (ver antes), vistas lateral, dorsal, ventral y anterior. d1-d3: DPO 33404, mismo yac. anterior, vistas anterior, lateral y dorsal. f1-f4: DPO 34095, mismo yac. anterior, vistas ventral, anterior, dorsal y lateral. g1g4: DPO 33421, procedente del valle alto del arroyo de El Cable (área de Gildar-Montó, S de Posada de Valdeón, León), cerca de una cabaña de cazadores, unos metros por debajo del Mb. Man, Eifeliense superior, vistas lateral, ventral, dorsal y anterior. e1-e3: Palentiella willefertae (Drot, 1971) DPO 33427, procedente de Marruecos, Oui Halan (=Tamjout-Ouihlane, en Drot, 1971), yac. Tm. 126 de Henri Hollard, Eifeliense superior, vistas ventral, anterior y dorsal. Barras negras: $1 \mathrm{~cm}$ (la inferior correspondiente a las figuras d1-d3 y la superior al resto de figuras).

$\boldsymbol{a}-\boldsymbol{d}$ and $\boldsymbol{f}$-g: Palentiella palentina n.g. n.sp. a1-a4: DPO 25171 (new picture of specimen figured as "Camarotoechia" willefertae Drot, in García-Alcalde et al., 1990, fig. 9.1-4, additionally enlarged), locality $\alpha-263$ (see above), ventral, dorsal, anterior, and lateral views. b1-b4: Paratype DPO 33417, loc. $\alpha$-49bis (see above), lateral, ventral, dorsal, and anterior views. c1-c4: DPO 33411, loc. $\alpha$-263 (see above), lateral, dorsal, ventral, and anterior views. d1-d3: DPO 33404, same loc. as before, anterior, lateral, and dorsal views. f1-f4: DPO 34095, same loc. as before, ventral, anterior, dorsal, and lateral views. g1-g4: DPO 33421, El Cable Creek upper valley (Gildar-Montó area, S of Posada de Valdeón, León), near a hunter shelter, some meters below the Man Mb sandstones, upper Eifelian, lateral, ventral, dorsal, and anterior views. e1-e3: Palentiella willefertae (Drot, 1971) DPO 33427, from Morocco, Oui Halan (=Tamjout-Ouihlane, in Drot, 1971), Henri Hollard's loc. Tm. 126, upper Eifelian, ventral, anterior, and dorsal views. Black bars: 1 cm (lower bar corresponding to figs. d1-d3; upper bar: the rest of figures). 


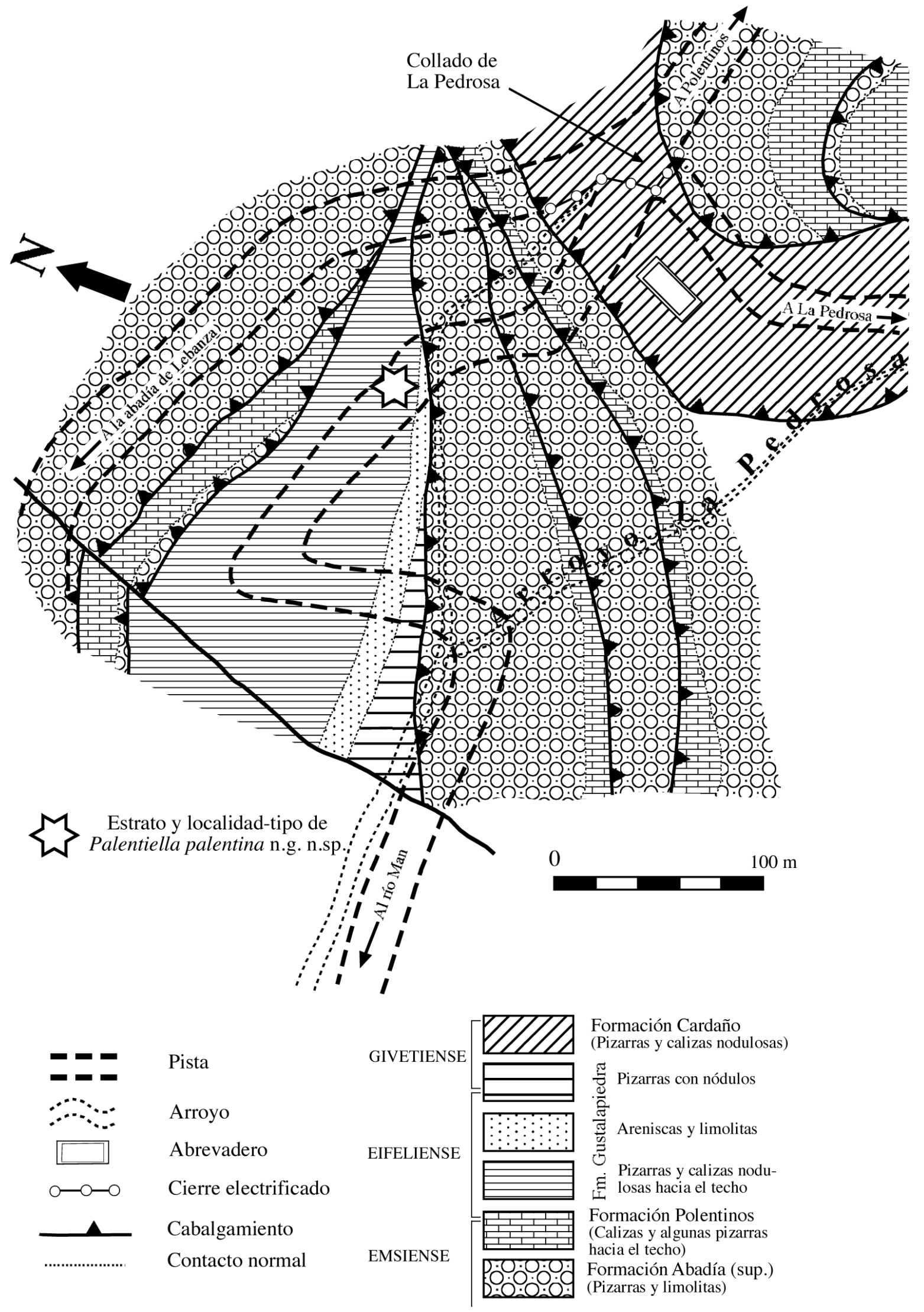

Figura 9. Situación geográfica y geológica de la localidad tipo de Palentiella palentina n.g. n.sp, 1,2 km al ONO del pueblo de Polentinos (N Cervera de Pisuerga, Palencia).

Geological and geographical setting of the Palentiella palentina n.g. n.sp. type-locality, $1.2 \mathrm{~km}$ WNW Polentinos village (N Cervera de Pisuerga, Palencia). 

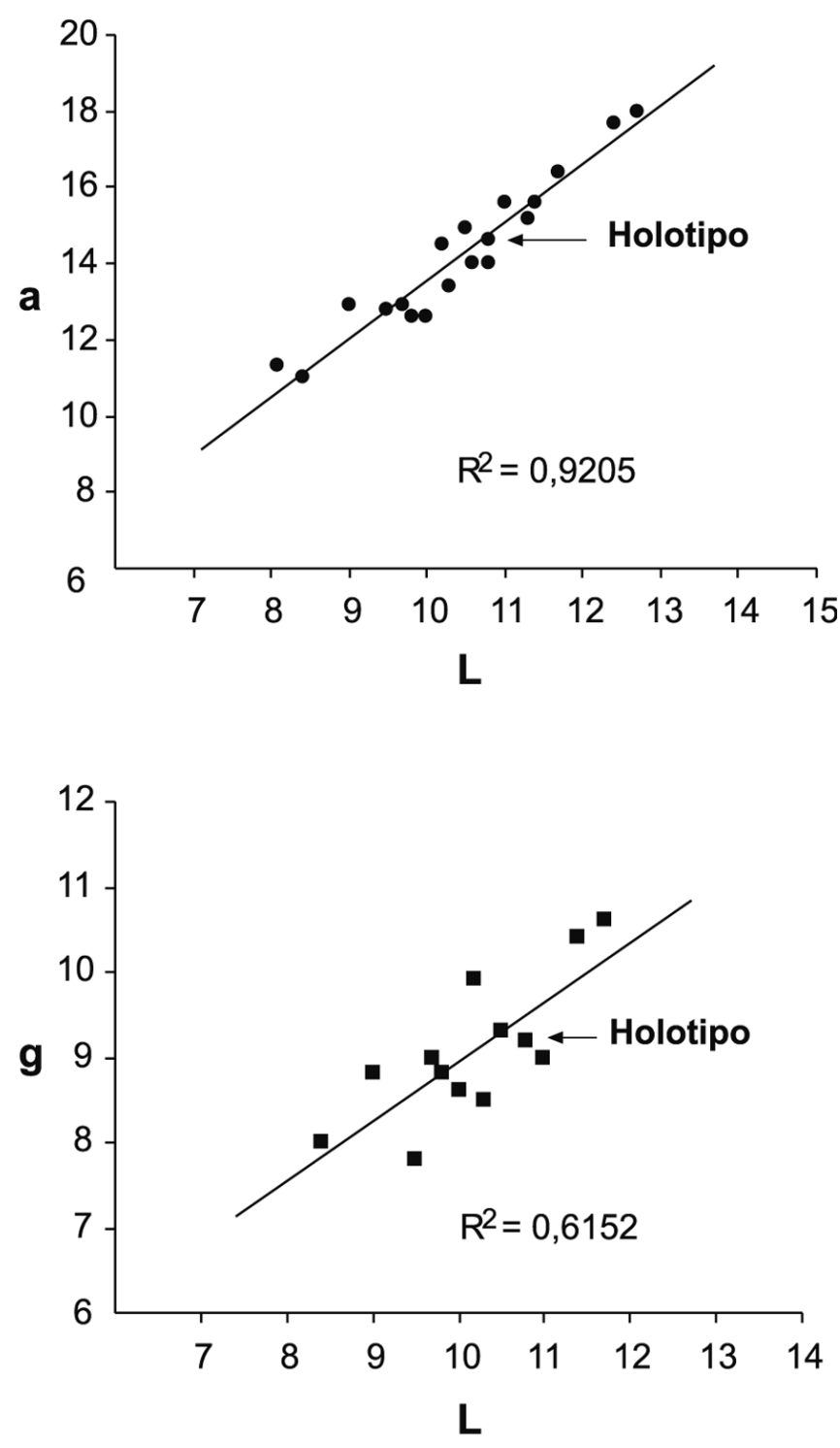

Figura 10. Palentiella palentina n.g. n.sp. Diagramas de dispersión, Longitud/anchura (L/a) y Longitud/grosor (L/g). Palentiella palentina $n . g . n . s p$. Length/width $(L / a)$ and Length/thickness $(\mathrm{L} / \mathrm{g})$ dispersion diagrams.

Campo muscular dorsal y otras huellas del sarcoglifo, desconocidas.

Placas dentales menudas, no funcionales, casi inexistentes (Figs. 11 y 12). Dientes cardinales relativamente pequeños, denticulados, extendiéndose hacia delante hasta el borde anterior de la plataforma cardinal; cavidad umbonal media profunda, alojando el extremo posterior de la otra valva. En el interior de la valva dorsal hay una plataforma cardinal entera, indivisa, deprimida en su parte media, soportada proximalmente por un septo medio alto y delgado, que se extiende por delante de la plataforma cardinal hasta más allá de la mitad de la longitud, definiendo cavidades apicales laterales dorsales libres, bien desarrolladas. En la parte proximal de la plataforma, se desarrolla una apófisis cardinal fuerte, relativamente alta, pectinada, que termina antes del frente de la plataforma (Fig. 12). Crura sub-

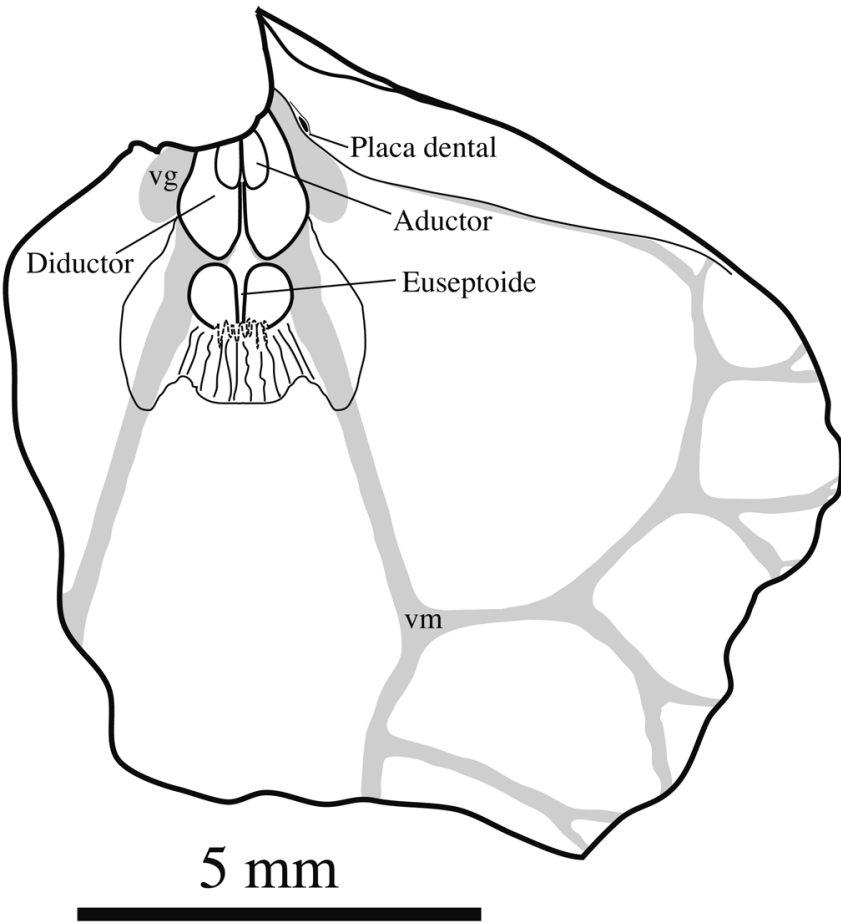

Figura 11.Dibujo del interior ventral de Palentiella palentina n.g. n.sp. basado en el ejemplar DPO 33403 (Fig. 7c). En gris: angioglifo; vg: vascula genitalia; vm: vascula media.

Drawing of Palentiella palentina n.g.n.sp, ventral interior. Based on specimen DPO 33403 (Fig. 7c) Grey colour: mantle canal system; vg: vascula genitalia; vm: vascula media.

cilíndricos, próximos, extendiéndose hacia delante en el plano de la comisura 1/3 o más de la longitud de la concha, con extremos distales proyectándose débilmente en dirección ventral.

Discusión: García-Alcalde (en García-Alcalde et al., 1990) comparó la nueva especie con "Camarotoechia" willefertae Drot, 1971 (=Palentiella willefertae). La forma marroquí tiene ornamentación, contorno y estructuras internas próximas a las de $P$. palentina, como cabe esperar de una especie del mismo género, pero se diferencia fácilmente en que es más transversa, mucho más baja, con la máxima altura en el tercio posterior, débilmente inequivalva, con invaginación frontal menos acusada, comisuras cortantes y número algo menor de costillas radiales. La especie africana tiene probablemente la misma edad que la nuestra, Eifeliense superior, aunque su autora la asignó a la transición Eifeliense-Givetiense, basándose en su asociación con Cabrieroceras crispiforme (Drot, 1971: 90). Formas de Cabrieroceras comparables con C. crispifor$m e$, en efecto, se asocian con formas de P. palentina, por debajo del Mb. Man de la Fm. Gustalapiedra, en toda la región palentina (Fig. 2). 


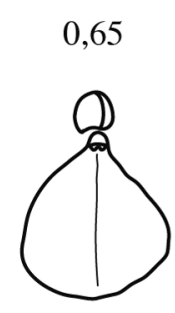

1,5

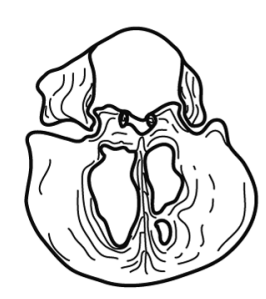

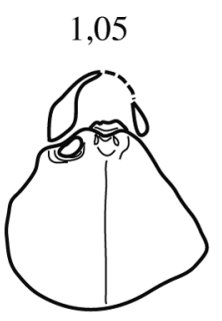

1,55

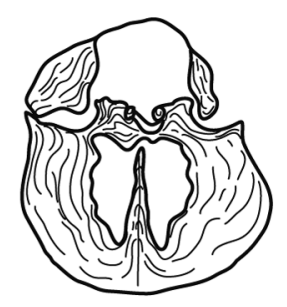

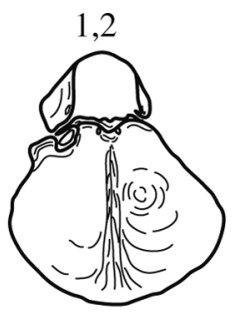

1,95

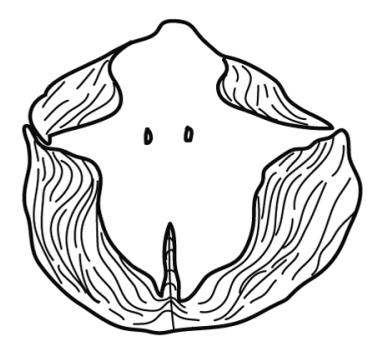

a

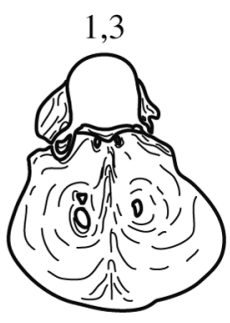

2,1

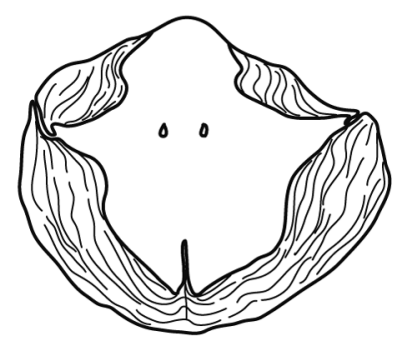

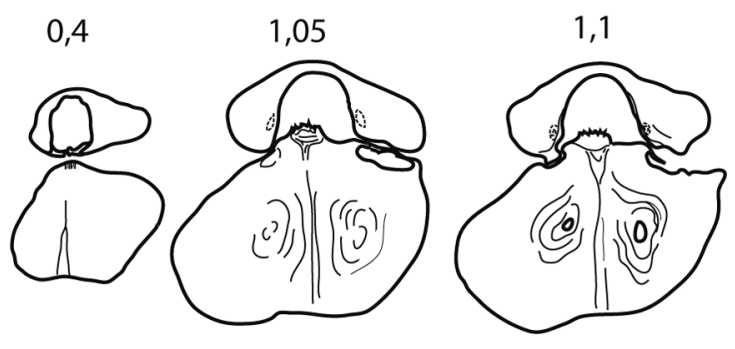
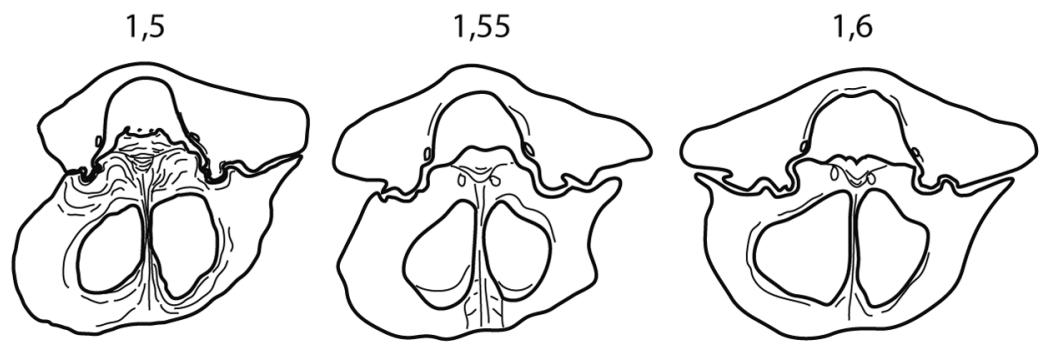

1,7
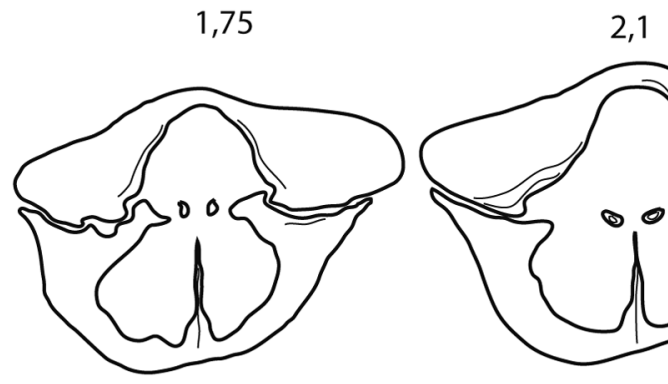

2,9
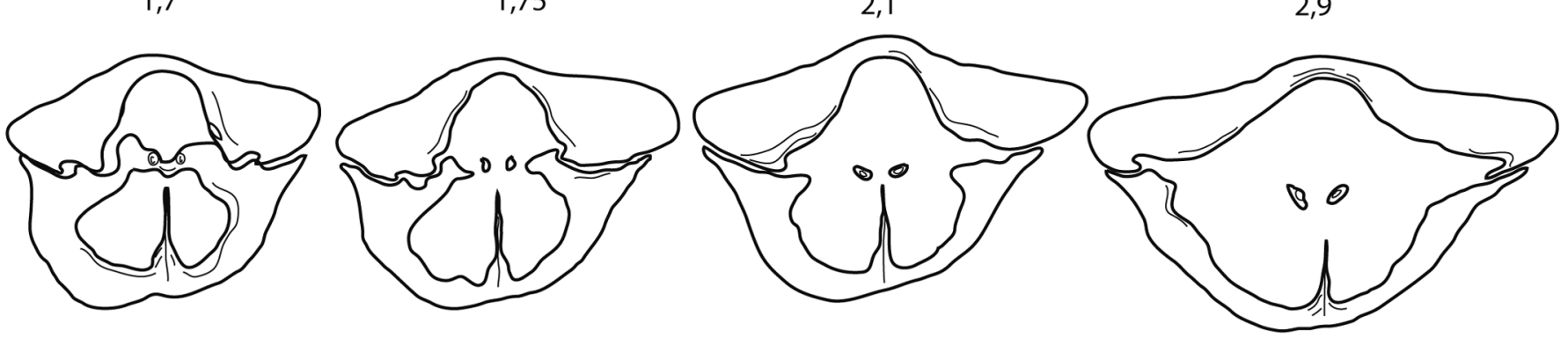

3,15

3,4

3,6
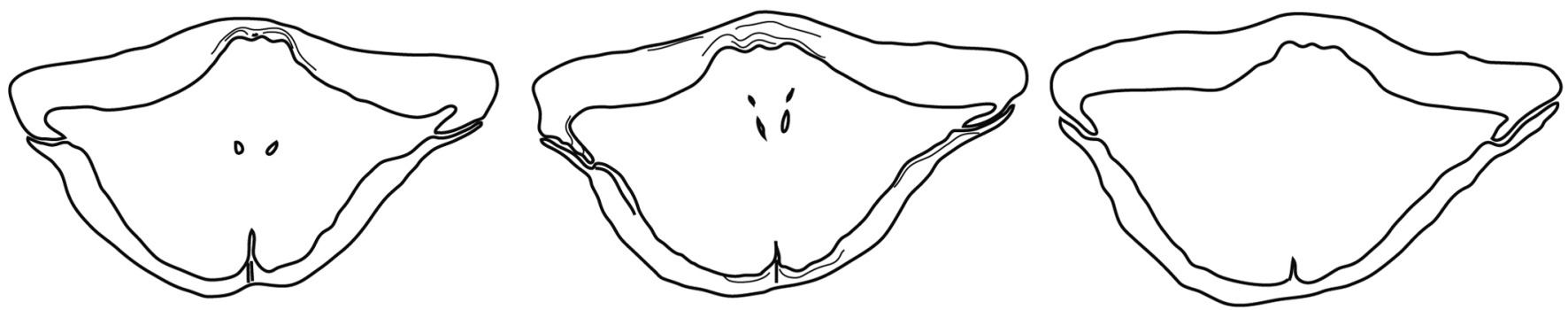

b

Figura 12. Palentiella palentina n.g. n.sp. Secciones seriadas de los ejemplares DPO 33402 (a) y DPO 34090 (b). Barra 0,5 mm. Palentiella palentina n.g.n.sp. Serial sections of specimens DPO 33402 (a), and DPO 34090 (b). Scale bar 0.5 mm. 
Palentiella hertae (Havlíček, 1961), una forma bohémica del Eifeliense superior, es también similar a la nuestra en perfil, contorno, ornamentación y estructura interna, pero es más alargada, bastante más baja, con ángulo apical menos obtuso, e invaginación frontal casi inexistente.

Distribución geográfica y estratigráfica: P. palentina n.g. n.sp. es una forma escasa pero omnipresente en todos los afloramientos de la Fm. Gustalapiedra, del área Palentina (Figs. 3 y 9), algo por debajo del Miembro Man de la referida formación (Fig. 2), en calizas nodulosas, alternando con pizarras negras. Su edad, de acuerdo con los escasos y dispersos datos de conodontos, sería Eifeliense superior (Biozona de T. kockelianus).

\section{CONCLUSIONES}

Entre las raras formas de braquiópodos bentónicos del Devónico Medio del Dominio Palentino, destaca por su carácter ubicuo, aunque representada siempre por un corto número de individuos, Palentiella palentina, un nuevo género y especie de camarotoéquido septalárido. Esta forma se acantona en capas del Eifeliense superior, por debajo de las areniscas del Miembro Man de la Formación Gustalapiedra y constituye una excelente guía estratigráfica en el dominio facial Palentino de la Cordillera Cantábrica oriental. El nuevo género comprende otras dos especies, también escasas: P. willefertae (Drot, 1971), de Marruecos, y P. hertae (Havlíček, 1961), de la República Checa, previamente incluidas en los géneros "Camarotoechia" y Nemesa, respectivamente. Las especies de Palentiella son todas del Eifeliense superior, propias de ambientes de plataforma externa relativamente profunda, de la Magnafacies Herciniana (o Bohemo-Herciniana). El origen del género y de sus especies se encontraría en la fase de renovación faunística que siguió al Evento Choteč-jugleri (Eifeliense inferior), pero no llegaron a superar el Evento Kačákotomari (Eifeliense terminal).

\section{AGRADECIMIENTOS}

Este trabajo forma parte del proyecto PICG 499 "Evolución de los ecosistemas y climas durante el Devónico" y fue financiado por el proyecto MEC-05-CGL 2005-03715 "Fases de desarrollo de asociaciones de corales y estromatoporoideos en el Devónico inferior de la Cordillera Cantábrica (NO de España) y relación de estos sucesos con eventos geo-biológicos" y Fondos FEDER. Se agradece al Servicio Territorial de Medio Ambiente de la Junta de Castilla-León el permiso y las facilidades para trabajar dentro de la Reserva Natural de Fuentes Carrionas, en Palencia. Mi más sincero reconocimiento a D. Francisco Juan Cabo del Olmo, "Quico", de Cervera de Pisuerga, por su experta orientación en los problemas administrativos y de desplazamiento por la Reserva Natural y sus aledaños. Expresivas gracias a los revisores, Profs. Miguel V. Pardo y Peter Carls, que mejoraron substancialmente el trabajo original.

\section{BIBLIOGRAFÍA}

Barrande, J. 1879. Système Silurien du centre de la Bohême. lère partie: Recherches paléontologiques, vol. 5: Classe des Mollusques. Ordre des Brachiopodes. Praga-París, $226 \mathrm{pp}$.

Brouwer, A. 1964. Deux faciès dans le Dévonien des Montagnes Cantabriques méridionales. Breviora Geológica Astúrica, 8, 3-10.

Drot, J. 1971. Rhynchonellida siluriens et dévoniens du Maroc présaharien. Nouvelles observations. Notes du Service Géologique du Maroc, 31, 65-108.

Erben, H.K. 1962. Zur Analyse und Interpretation der rheinischen und hercynischen Magnafacies des Devons. In: Internationale Arbeitstagung über die Silur/Devon-Grenze und die Stratigraphie von Silur und Devon, Bonn/Bruxelles 1960. E. Schweizerbart'sche Verlagsbuchhandlung, Stuttgart, 2, 42-61.

García-Alcalde, J.L. 1996. El Devónico del dominio AsturLeonés en la Zona Cantábrica ( $\mathrm{N}$ de España). Revista Española de Paleontología, $\mathbf{n}^{\mathbf{0}}$ extraordinario, 58-71

García-Alcalde, J.L. 1998a. Braquiópodos rinconélidos del Lochkoviense (Devónico Inferior) de la Cordillera Cantábrica (Norte de España). Geobios, 31, 767-789.

García-Alcalde, J.L. 1998b. Devonian events in northern Spain. Newsletter in Stratigraphy, 36, 157-175.

García-Alcalde, J.L. 1999. Braquiópodos hercínicos del Eifeliense superior (Devónico Medio) del Dominio Palentino (N de España). Temas Geológico-Mineros ITGE, 26, 599-604.

García-Alcalde, J.L., Montesinos, J.R., Truyóls-Massoni, M., García-López, S., Arbizu, M.A. \& Soto, F. 1988. El Silúrico y el Devónico del Dominio Palentino (NO de España). Revista de la Sociedad Geológica de España, 1, 7-13.

García-Alcalde, Arbizu, M., García-López, S., Leyva, F., Montesinos, R., Soto, F. \& Truyols-Massoni, M. 1990. Devonian stage boundaries (Lochkovian/Pragian, Pragian/ Emsian, and Eifelian/Givetian) in the Cantabric region (NW Spain). Neues Jahrbuch Geologie und Paläontologie Abhandlungen, 180, 177-207.

García-Alcalde, J.L. (coordinator), Carls, P., Pardo Alonso, M.V., Sanz López, J., Soto, F., Truyols-Massoni, M. \& Valenzuela-Ríos, J.I. 2002. Devonian. In: The Geology of Spain (eds. W. Gibbons \& T. Moreno). The Geological Society, London, 67-91.

Havlíček, V. 1960. Bericht über die Ergebnisse der Revision der Böhmischen Altpaläozoischen Rhynchonelloidea. Věstník Ústredního ústavu geologického, 35, 241-244.

Havlícek, V. 1961. Rhynchonelloidea des böhmischen älteren Paläozoiku ms (Brachiopoda). Vydal Ústřední ústav geologický, 27, 1-211. 
Jahnke, H., Henn, A., Mader, H. \& Schweineberg, J. 1983. Silur und Devon im Arauz-Gebiet (Prov. Palencia, NSpanien). Newsletter in Stratigraphy, 13, 40-66.

Leidhold, C. 1928. Beiträg zur Kenntnis der Fauna des rheinischen Stringocephalenkalkes, insbesondere seiner Brachiopodenfauna. Abhandlungen der preussischen geologischen Landesanstalt, N.F, 109, 1-99.

Prado, C. de \& Verneuil, E. de 1850. Note géologique sur les terrains de Sabero et de ses environs dans les montagnes de Léon (Espagne), suivie d'une description des fossiles de ces terrains. Bulletin de la Société Géologique de France, $2^{\text {eme. }}$ ser. 7, 137-186.

Schmidt, H. 1941. Die mitteldevonischen Rhynchonelliden der Eifel. Abhandlungen der Senckenbergischen Naturforschenden Gesellschaft, 459, 1-79.

Schubert, C. 1929. Classification of brachiopod genera, fossils and Recent. In: Brachiopoda (Generum et Genotyporum Index et Bibliographia) (C. Schubert \& C. M. Levene). Fossilium Catalogus I: Animalia, 42, 10-25.
Truyols-Massoni, M., Montesinos, R., García-Alcalde, J.L. \& Leyva, F. 1990. The Kačák-Otomari event and its characterization in the Palentine Domain (Cantabrian Zone, NW Spain). In: Extinction events in Earth History (eds. E.G. Kauffman \& O.H. Walliser). Lecture Notes in Earth Sciences, 30, 133-143.

Verneuil, E. de \& d'Archiac, V. (1845). Note sur les fossiles du terrain paléozoique des Asturies. Bulletin de la Société Géologique de France, $2^{\text {eme. }}$ ser. 2, 458-480.

Walliser, O.H. 1985. Natural boundaries and Commission boundaries in the Devonian. Courier Forschungsinstitut Senckenberg, 75, 401-408.

Wedekind, R. 1926. Die Devonische Formation. In: Grundzüge der Geologie, Band II: Erdgeschichte (W. H. Salomon). E. Schweizerbart'sche Verlagsbuchhandlung, Stuttgart, 194-226.

Manuscrito recibido: 3 de Julio, 2008 Manuscrito aceptado: 11 de Mayo, 2009 